\title{
Geometric Systems of Disjoint Representatives ${ }^{\star}$
}

\author{
Jiří Fiala ${ }^{1}$, Jan Kratochvíl ${ }^{1, \star \star}$, and Andrzej Proskurowski ${ }^{2, \star \star \star}$ \\ 1 Institute for Theoretical Computer Science ${ }^{\dagger}$ \\ and Department of Applied Mathematics, \\ Charles University, Prague \\ \{fiala, honza\}@kam.mff.cuni.cz \\ 2 Department of Computer and Information Science, \\ University of Oregon, Eugene \\ andrzej@cs. uoregon.edu
}

\begin{abstract}
Consider a finite collection of subsets of a metric space and ask for a system of representatives which are pairwise at a distance at least $q$, where $q$ is a parameter of the problem. In discrete spaces this generalizes the well known problem of distinct representatives, while in Euclidean metrics the problem reduces to finding a system of disjoint balls. This problem is closely related to practical applications like scheduling or map labeling. We characterize the computational complexity of this geometric problem for the cases of $L_{1}$ and $L_{2}$ metrics and dimensions $d=1,2$. We show that for $d=1$ the problem can be solved in polynomial time, while for $d=2$ we prove that it is NP-hard. Our NP-hardness proof can be adjusted also for higher dimensions.
\end{abstract}

\section{Introduction}

Take a universe $X$ and a family of its subsets $\mathcal{M}=\left\{M_{i} \mid i \in I, M_{i} \subseteq X\right\}$. The System of Distinct Representatives (SDR) for $\mathcal{M}$ selects from each $M_{i}$ an element, such that sets are represented by distinct elements. Such assignment can be formally described by an injective mapping $f: I \rightarrow X$ satisfying the property $f(i) \in M_{i}$ for all $i \in I$.

Observe that such system can be equivalently formulated as a matching in bipartite graph, where vertices of the first block of the bi-partition correspond to the elements of $X$, the other vertices stand for the sets $M_{i}$ and edges describe the incidence relation.

The theory of Systems of Distinct Representatives is well known and very important for discrete optimization problems. Namely, in the case when the

* The authors acknowledge support of joint Czech U.S. grants KONTAKT ME338 and NSF-INT-9802416 during visits of the first two authors to Eugene, OR, and of the third author to Prague.

** Research partially supported by Czech Research grant GAUK 158/99.

$\star \star \star$ Supported in part by the grant NSF-ANI-9977524.

† Supported by the Ministry of Education of the Czech Republic as project LN00A056. 
family $\mathcal{M}$ and all its sets $M_{i}$ are finite, the elegant Hall theorem [5] describes necessary and sufficient conditions for the existence of an SDR, and if it exists it can be found by a polynomial time algorithm (either the algorithm involving augmenting paths or the matching algorithm of Edmonds [3]).

Though several generalizations of the concept have been studied, we believe that the concept of distant representatives is new. We assume that the universe $X$ is equipped by a metric $\operatorname{dist}(a, b)$ and therefore we may ask for representatives that are sufficiently spaced in $X$. More formally:

Definition 1. Given a parameter $q>0$ and a family $\mathcal{M}=\left\{M_{i} \mid i \in I\right\}$ of subsets of $X$, a mapping $f: I \rightarrow X$ is called a System of $q$-Distant Representatives $(S q-D R)$ if

(1) $f(i) \in M_{i}$ for every $i \in I$,

(2) $\operatorname{dist}(f(i), f(j)) \geq q$ for every $i, j \in I, i \neq j$.

The metric on the space $X$ could be defined in several ways, for example:

- The trivial metric: For arbitrary universe $X$ we set $\operatorname{dist}(a, b)=0$ if $a=b$, and $\operatorname{dist}(a, b)=1$ otherwise.

- The integral metric: $X=\mathbb{N}$, $\operatorname{dist}(a, b)=|a-b|$.

- The graph metric: Take $X=V(G)$ and set $\operatorname{dist}(a, b)$ to the length of the shortest path connecting vertices $a$ and $b$ in a graph $G$.

- The plane Euclidean metric: $X=\mathbb{R}^{2}$, distance is the length of the segment joining the points $a$ and $b$ in the plane.

- The plane Manhattan distance is defined as the sum of coordinate differences $\left|a_{x}-b_{x}\right|+\left|a_{y}-b_{y}\right|$. The universe $X=\mathbb{R}^{2}$ as in the previous case.

Observe that in the case of trivial or integral or graph metric the System of $q$-Distant Representatives is equivalent to System of Distinct Representatives as far as $q \leq 1$. This holds in slightly more general setting: Consider any universe $X$ equipped by any metric for which $\operatorname{dist}(a, b) \geq 1$ whenever $a \neq b$. Then for $q \leq 1$ the condition (2) merely says that $f(i) \neq f(j)$, i.e., a System of $q$-Distant Representatives is a System of Distinct Representatives. For this case any further metric structure on $X$ becomes irrelevant and in such a case an $\mathrm{S} q$-DR could be found by a polynomial-time algorithm.

We address the computational complexity of the Sq-DR problem in [4] and show several NP-hardness results, mainly for the discrete spaces. Some cases provide a natural counterpart to the results of Aharoni and Haxell [1] who studied a more general concept of Systems of Disjoint Representatives on Hypergraphs. Though they derive necessary and sufficient conditions for the existence of such system on finite families $\mathcal{M}$, the problem whether these conditions are satisfied is still $N P$-hard as we show in [4].

The aim of this paper is to show that for unit balls in the Euclidean plane the S1-DR problem is NP-hard for both Euclidean an Manhattan metrics. This is in contrast with the complexity of the corresponding $\mathrm{S} q$-DR problem for balls in the one dimensional Euclidean space, where it allows a polynomial-time algorithm. 


\section{Systems of Distant Representatives in the Euclidean Space}

In this section we consider the case when the universe $X$ is the $d$-dimensional Euclidean space $\mathbb{R}^{d}$ equipped with the $L_{p}$ metric for some fixed $p$. (The $L_{p}$ metric is defined by the formula: $\left.\operatorname{dist}_{L_{p}}(a, b)=\sqrt[p]{\sum_{j=1}^{d}\left|a_{j}-b_{j}\right|}\right)$. Clearly, the collection $\mathcal{M}$ consists of sets of points. If all sets $M_{i}$ are finite, we can transform such instance to a finite graph $G$ (here vertices represent points, and pairs of them are adjacent when the corresponding points are at distance less than $q$ ) and look for 2-distant representatives in the graph metric. We omit for the moment this case and focus our attention on infinite sets $M_{i}$, namely unit diameter balls in $\mathbb{R}^{d}$. From the computational complexity it is important that such infinite sets can be described in constant space, e.g. by the coordinates of the centers of the balls.

Observe that in any $L_{p}$ metric, two points $a$ and $b$ are at distance smaller than $q$, iff the two open balls of diameter $q$, one centered at $a$ and the other at $b$, intersect. The S $q$-DR problem for closed unit balls in $\mathbb{R}^{d}$ then has a nice geometric representation: replace each closed unit ball $M_{i}$ of $\mathcal{M}$ by an open ball $M_{i}^{\prime}$ of diameter $1+q$ and ask, whether each $M_{i}^{\prime}$ can be assigned as a representative a ball of diameter $q$, completely placed inside $M_{i}^{\prime}$, all these representatives being pairwise disjoint. In the rest we use this representation $\mathcal{M}^{\prime}$ of the $\mathrm{S} q$-DR problem, rather than the original collection $\mathcal{M}$. The reason is that in this representation we do not need to operate with the underlying distance: all essential properties are captured by the inclusion relation. A similar situation was considered for finite sets by Aharoni and Haxell in [1].

It is clear that such problems can naturally arise in practical applications like map labeling (for $d=2$ ), where the sets $M_{i}$ correspond to the possible label placement, and the disjoint representatives of diameter $q$ correspond to the reserved places for labels. See [7,2] for hardness results on similar map labeling problems.

The one-dimensional version of finding an $\mathrm{S} q$-DR for (unit) balls in $\mathbb{R}$ is equal to the following scheduling problem: Let a set $I$ of tasks be given. These tasks require to be scheduled on one processor without preemption, such that each task is processed for the same time $(q)$ and within the predetermined time bounds - the release date and the due date - that form intervals $\left(r_{i}, d_{i}\right)\left(M_{i}=\right.$ $\left.\left(r_{i}+\frac{q}{2}, d_{i}-\frac{q}{2}\right)\right)$. The question is whether a feasible schedule exists.

Clearly, the polynomial-time algorithm solving the scheduling problem due to Simons 8] provides also a solution for the $\mathrm{S} q$-DR problem on $\mathbb{R}$ with any $L_{p}$ metric (the parameter $p$ is irrelevant in the one-dimensional Euclidean space). We show in the next section that in the two-dimensional space (and hence in higher dimensions as well) the problem becomes $N P$-hard for both the Euclidean $\left(L_{2}\right)$ and the Manhattan $\left(L_{1}\right)$ metrics. 


\section{Systems of Distant Representatives in the Plane}

In the Euclidean metric, balls correspond to disks, while in the Manhattan metric we get squares of diagonal length equal to the required diameter, and diagonals are parallel with the coordinate axes. For convenience, in the case of the Manhattan metric we consider that the plane and all its objects are rotated by $\frac{\pi}{4}$ and scaled by the factor $\sqrt{2}$, hence all squares involved have side length $1+q$ (in $\mathcal{M}^{\prime}$ ) or $q$ (the representatives), and squares are oriented in the usual way.

Theorem 1. The S1-DR problem is NP-hard for closed unit squares in the Manhattan metric.

Proof. We show a reduction from the planar 3-SAT problem, whose NP-completeness was proved in [6]. An instance for this version of the satisfiability problem consists of a formula $\Phi=(V, C)$ in the conjunctive normal form, where each variable has one positive and two negative occurrences, each clause consists of two or three literals, and the incidence graph $G$ of $\Phi$ is planar. The graph $G$ has vertex set $V(G)=V \cup C$ and an edge $(x, c)$ belongs to $E(G)$ iff the clause $c$ contains $x$ or $\neg x$ as a literal.

The family $\mathcal{M}^{\prime}$ contains squares of side length 2 . It can be split into three parts: one part represents clauses, the next variables, and the third part are connectors joining clause and variable gadgets together.

The three-literal clause gadget consists of two squares of the same placement. It is clear, that only two of the four quadrants could be completely free whenever an S1-DR exists. Similarly, for a clause with only two literals we use three squares in the same position.

The variable gadget is schematically depicted in Fig. 1. The dashed lines represent the unit grid in the plane. By black lines we depict 16 squares which we call active, the bold lines indicate two squares at the same position. The halftoned area cannot be used for a possible representative of an active square - it is fully occupied e.g. by four squares at the same position (hence each of them is represented by a different quadrant).

The important property of the variable gadget is, that if any S1-DR exists, then either squares $P_{1}$ and $P_{2}$ or $N_{1}$ and $N_{2}$ will be partly occupied by the representatives for the vertex gadget. For example, if neither $P_{1}$ nor $P_{2}$ is hit by the representatives, then both $N_{1}$ and $N_{2}$ are, as depicted in Fig. 2. The small squares are representatives for the active squares of the gadget, their numbering corresponds to left-to-right ordering of the active squares from Fig. 1.

The connector gadget corresponds to a chain of squares. These squares are grouped to triples and all squares in a triple have the same position (so Fig. 3 represents in fact 18 squares). Two consecutive triples in the chain share one quadrant. If we select two quadrants at the ends of the chain, like $A$ and $B$ in the figure, then at least one of them is fully occupied, if the chain has an S1-DR.

The collection $\mathcal{M}^{\prime}$ contains for each clause $c \in C$ a disjoint copy of the clause gadget placed on a grid according to the planar drawing of $G$. Similarly we place the variable gadgets for each variable $x \in V$. If a variable $x$ occurs positively 


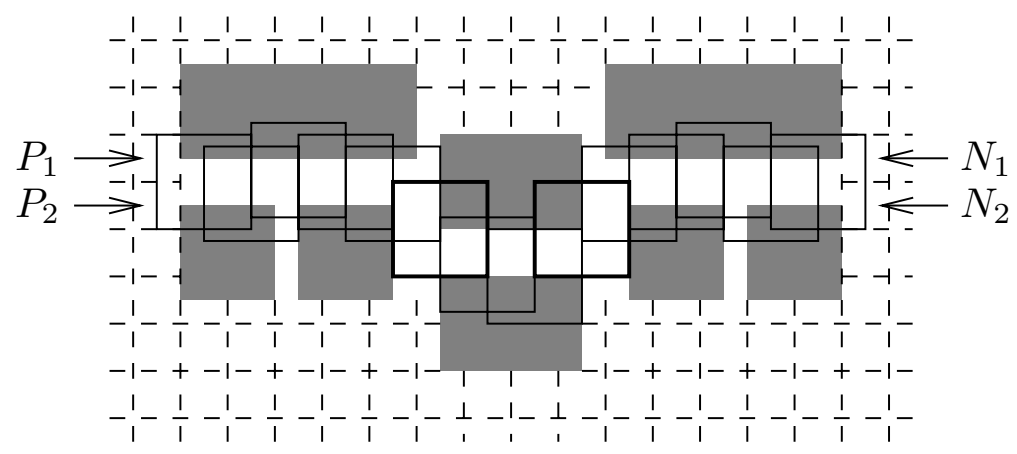

Fig. 1. Variable gadget.

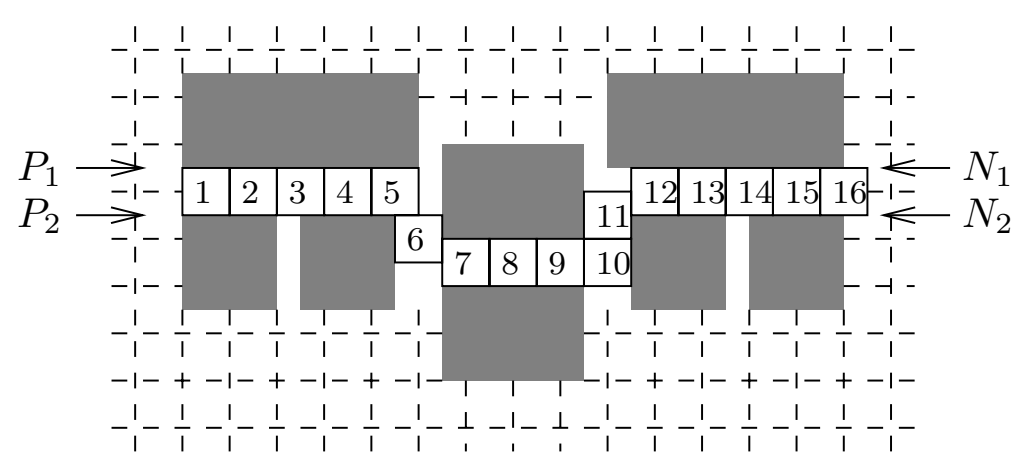

Fig. 2. S1-DR for the variable gadget corresponding to the truth assignment.

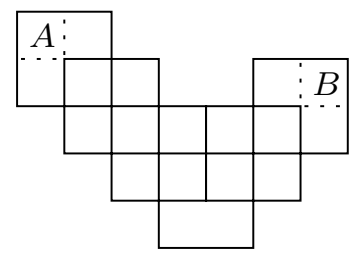

Fig. 3. Connector gadget.

in a clause $c$, we include into $\mathcal{M}^{\prime}$ a connector joining a unique quadrant of the clause gadget representing $c$ with a lower right quadrant of the square $P_{1}$ related to the variable gadget of $x$. We similarly use left corners of $N_{1}$ and $N_{2}$ for the two (possible) occurrences of $\neg x$.

The placement of all these objects in the plane follows the planar drawing of the graph $G$ on the unit grid. All squares except those in the construction of the variable gadget coincide with the grid, so the entire collection $\mathcal{M}$ can be constructed such that its size is polynomial in the size of the formula $\Phi$. 
Assume that an S1-DR for $\mathcal{M}^{\prime}$ exists. No clause gadget cannot serve all its literals by a free quadrant, hence at least one these literals is forced to fully occupy the square $P_{1}$ (for a positive literal) or some $N_{i}$ (otherwise) of the corresponding variable gadget. As it was mentioned above, it is impossible to occupy both $P_{1}$ and some $N_{i}$ of the same gadget at the same time. Hence, we may define an assignment $x=$ true if $P_{1}$ is not occupied by the representatives for the active squares of the variable gadget for $x$ and we put $x=$ false otherwise. Such assignment is well defined and satisfies $\Phi$.

The construction of an S1-DR from a truth assignment of $\Phi$ is then straightforward.

In the proof of Theorem 1 only few squares were placed out of the unit grid. We show, that in the case if all unit squares respect the grid, than the problem is solvable by a polynomial-time algorithm.

Corollary 1. For any $q$ of form $\frac{1}{k}, k \in \mathbb{N}$ it holds that the Sq-DR problem for closed unit squares can be solved in polynomial time, if all sides of squares in $\mathcal{M}$ coincide with the grid of span $q$.

Proof. If any $\mathrm{S} q$-DR exists, then shift all the representatives to the left\&downmost position. The resulting Sq-DR has the property that all representatives coincide with the grid points.

Then we can without loss of generality restrict the universe $X$ only to the grid points and solve the problem by finding a System of Distinct Representatives.

We conclude this section by proving an analogous result for disks, i.e., for the Euclidean metric.

Theorem 2. The S1-DR problem is NP-hard for unit disks in the Euclidean metric.

Proof. The proof mimics the proof of the case of squares. We represent a planar formula $\Phi$ by a collection of disks that are partitioned into clause and variable gadgets, and connectors. As above we use the representation $\mathcal{M}^{\prime}$, where representatives correspond to disks of diameter 1 , while the disks in $\mathcal{M}^{\prime}$ have diameter 2 .

The first tool involved in the construction is the reserved halftoned area. Observe that the four disks depicted in Fig. 4 a) allow an S1-DR only as the four halftoned disks.

The connectors are depicted in Fig. 4 b). In no S1-DR of the connector gadget both disks $A$ and $B$ may remain simultaneously empty. The vertex gadget is illustrated in the part c). As in the previous proof, it is impossible to find representatives for the vertex gadget where some $P_{i}$ remains free together with some $N_{j}$. Similarly, if the disk $P_{1}$ remains empty, the corresponding variable will be assigned the true value.

Finally, clause gadgets are shown in the section d). At least one of the three or two, resp., dashed disks cannot be left free to saturate the adjacent connector's end. 

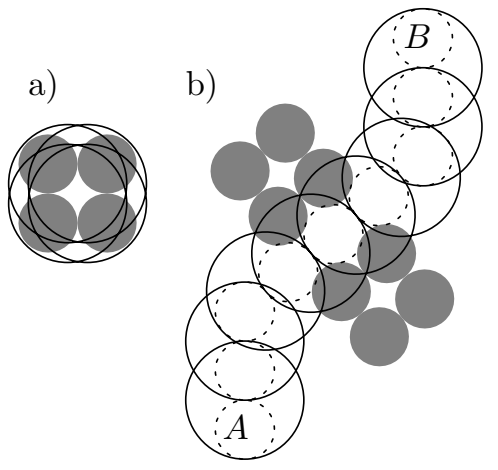

c)

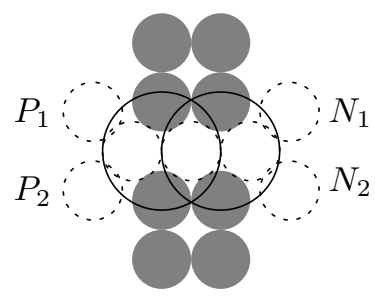

d)

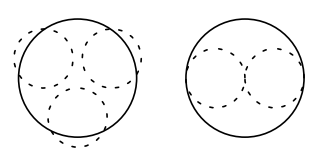

Fig. 4. Gadgets for the S1-DR problem on disks.

The entire discussion follows in the exactly same manner as in the case of squares.

\section{Conclusion}

In higher dimensions, the construction of connectors and variable and clause gadgets is similar as in the plane. We avoid it here due to space limitations and a somewhat technical aspect of the argument.

We also believe that both above constructions can be adjusted for other fixed values of $q<1$. We present as an open problem what is the computational complexity for the $\mathrm{S} q$-DR geometric problem with the Euclidean or Manhattan metrics for $q$ in the range $(1, \infty)$.

Finally, we believe that a uniform proof can be derived for the $N P$-hardness of the $\mathrm{S} q$-DR problem for unit balls in the $d$-dimensional Euclidean space for all $L_{p}$ metrics $(q>0, d \geq 2, p \geq 1)$,

It also remains as an open question whether the Sq-DR problem for unit balls belongs to the class NP. It is not straightforward to see whether the coordinates of the representatives - if they exist — can be described and verified in polynomial space and time.

\section{References}

1. Aharoni, R., P. Haxell, Hall's theorem for hypergraphs, J. Graph Th. 35, (2000), pp. 83-88.

2. Doddi S., M. V. Marathe, A. Mirzaian, B. M. E. Moret, B. Zhu, Map labeling and its generalizations. Proc. 8th ACM-SIAM Symp. on Discrete Algorithms, (1997), pp. $148-157$.

3. Edmonds, J., Paths, trees and flowers, Can. J. Math. 17, (1965), pp. 449-467.

4. Fiala, J., J. Kratochvíl, A. Proskurowski, Systems of sets and their representatives, manuscript 2002. 
5. Hall, P., On representatives of subsets, J. London Math. Soc. 10, (1935), pp. 2630 .

6. Kratochvíl, J., A special planar satisfiability problem and a consequence of its NP-completeness, Discrete Appl. Math. 52, (1994), pp. 233-252.

7. Marks, J., S. Shieber, The computational complexity of cartographic label placement, Technical Report TR-05-91, Harvard CS, 1991.

8. Simons, B., A fast algorithm for single processor scheduling, Proc. 19th Symp. Foundations of Computer Science, (1978), pp. 246-252. 